\title{
pH 值对共沉淀法制备钼基多金属氧化物催化异丁烯选择氧化 制甲基丙烯醛性能的影响
}

\author{
徐 达 ${ }^{1} \quad$ 刘士艳 $^{1} \quad$ 刘云凌 $^{2}$ 王振旅 ${ }^{1, *}$ \\ ('吉林大学化学学院, 吉林省表面与界面化学重点实验室, 长春 130021; \\ ${ }^{2}$ 吉林大学, 无机合成与制备化学国家重点实验室, 长春 130021)
}

\begin{abstract}
摘要：研究了共沉淀法制备 Mo-Bi-Fe-Co-Mn 五组分催化剂过程中溶液的 $\mathrm{pH}$ 值对催化剂中物种存在状态的 影响. 并考察其异丁烯选择氧化制备甲基丙烯醛(MAL)的反应性能. 结合粉末 X射线衍射 (XRD)、傅里叶变换红 外(FTIR)光谱、X射线光电子能谱(XPS)、氮气吸附-脱附和 $\mathrm{H}_{2}$ 程序升温还原 $\left(\mathrm{H}_{2}-\mathrm{TPR}\right)$ 等表征手段对催化剂的结 构进行了详细的研究. 结果表明, 溶液的 $\mathrm{pH}$ 值对催化剂结构和组成有明显的影响. 催化剂中某种特定物相 MX 随沉淀的 $\mathrm{pH}$ 值的提高而增加, 当 $\mathrm{pH}=7$ 时, 催化剂中 $\mathrm{MX}$ 相的量达最大值. 此时, 异丁烯的转化率和甲基丙烯䤊 的选择性分别为 $99.9 \%$ 和 $95.6 \%$. 进一步提高沉淀的 $\mathrm{pH}$ 值, 催化剂中 $\mathrm{MX}$ 相的量减少, 异丁烯的转化率和甲基 丙烯酲的选择性也相应降低. 研究表明, 催化剂上各组分间存在一定的相互作用, 催化剂中的特定物相 MX 是 影响反应活性的关键因素之一.
\end{abstract}

关键词：钼; 金属复合氧化物; $\mathrm{pH}$ 值; 异丁烯; 氧化 中图分类号: 0643.3

\section{Effect of pH Value on the Properties of Molybdenum-Based Multiphasic Oxide Catalysts in Selective Oxidation of Isobutene to Methacrolein}

\author{
XU Da ${ }^{1} \quad$ LIU Shi-Yan ${ }^{1} \quad$ LIU Yun-Ling ${ }^{2} \quad$ WANG Zhen-Lü1,* \\ ('Key Laboratory of Surface and Interface Chemistry of Jilin Province, College of Chemistry, Jilin University, \\ Changchun 130021, P. R. China; ${ }^{2}$ State Key Laboratory of Inorganic Synthesis and Preparative Chemistry, \\ Jilin University, Changchun 130021, P. R. China)
}

\begin{abstract}
The effect of different $\mathrm{pH}$ values on Mo-Bi-Fe-Co-Mn multiphasic oxide catalysts prepared via co-precipitation methods was studied. In addition, selective oxidation of isobutylene to methacrolein (MAL) reaction was evaluated. The structures and crystal phases of the catalysts were characterized by X-ray diffraction (XRD), Fourier transform infrared (FTIR) spectroscopy, X-ray photoelectron spectroscopy (XPS), $\mathrm{N}_{2}$ adsorption-desorption, and temperature-programmed reduction of $\mathrm{H}_{2}\left(\mathrm{H}_{2}-\mathrm{TPR}\right)$. The characterization results showed that the structures and properties depended on the $\mathrm{pH}$ during preparation. The amount of the special crystalline phase $\mathrm{MX}$ on the catalysts increased with increasing $\mathrm{pH}$ value during the preparation process. When the precipitation $\mathrm{pH}$ value was 7 , the crystalline phase $\mathrm{MX}$ reached maximum levels on the corresponding sample. The conversion of isobutene and selectivity for methacrolein were $99.9 \%$ and $95.6 \%$, respectively. When increasing the precipitation $\mathrm{pH}$, the $\mathrm{MX}$ content decreased, and the conversion of isobutene and selectivity for methacrolein decreased. The results revealed that synergistic effects or cooperation between multiphasic oxides and the special phase MX might be important for improving catalytic performance.
\end{abstract}

Received: June 21, 2012; Revised: August 22, 2012; Published on Web: August 28, 2012.

"Corresponding author. Email: wzl@jlu.edu.cn; Tel/Fax: +86-431-88499140.

The project was supported by the Open Subject of State Key Laboratory of Inorganic Synthesis and Preparative Chemistry, China (2012-15) and Innovation Project of Frontiers of Science and New Interdiscipline of Jilin University, China (2012).

无机合成与制备化学国家重点实验室开放课题(2012-15)及吉林大学科学前沿与交叉学科创新项目(2012)资助

(C) Editorial office of Acta Physico-Chimica Sinica 
Key Words: Molybdenum; Multiphasic oxides; $\mathrm{pH}$ value; Isobutene; Oxidation

\section{1 引言}

甲基丙烯酸甲酯(MMA)是重要的有机化工原 料. ${ }^{1,2}$ 异丁烯选择氧化制备甲基丙烯醛(MAL) 经氧 化、酯化制备 MMA 是一条有重要意义的工艺路线. ${ }^{3,4}$ 因此, 异丁烯选择氧化制备甲基丙烯醛反应越来越 受到人们的关注. ${ }^{5-11}$

人们对烯烃类选择氧化的催化剂体系进行了 大量的研究. ${ }^{9,12-15}$ 重点多为 Mo-Bi 基多元氧化物体 系. ${ }^{9}$ 由于催化剂组分多, 各组分间的相互作用非常 复杂, 对于活性中心的理解也各不相同. 例如, Van Well 等 ${ }^{16}$ 研究了 $\mathrm{Bi}_{2} \mathrm{MoO}_{6}$ 催化剂上丙烯选择氧化制 备丙烯醛反应, 结果发现焙烧温度对催化剂的性能 有很大影响. 低温短时间焙烧, 催化剂表面的 Bi/Mo 较低, 催化剂的活性高. 提高焙烧温度或延长焙烧 时间, $\mathrm{Bi}$ 物种在催化剂表面富集,催化活性降低. $\mathrm{JO}_{\mathrm{O}}$ 等 ${ }^{17}$ 研究了 $\mathrm{Mo}_{12} \mathrm{Bi}_{1.0} \mathrm{Co}_{4.4} \mathrm{Fe}_{1.0} \mathrm{~K}_{0.07} \mathrm{O}_{x}$ 催化剂上丙烯 氧化反应, 结果发现溶胶-凝胶法可制备小粒径的金 属氧化物催化剂,有效的提高反应活性. Le 等 ${ }^{18}$ 研究 了钼酸铋催化剂上丙烯氧化制备丙烯醛反应, 结果 发现当 $\mathrm{Bi} / \mathrm{Mo}$ 比为 1.3 且同时存在钼酸铋的 $\alpha$ 和 $\gamma$ 相 时, 氧化物之间存在协同作用, 反应活性高.

在异丁烯选择氧化反应中, 人们针对钼铋基多 组分催化剂也进行了大量的研究. 例如, Carrazán 等 ${ }^{19}$ 研究了钿酸铋催化剂上异丁烯氧化制备甲基丙 烯醛反应. 结果发现, 复合氧化物 $\gamma-\mathrm{Bi}_{2} \mathrm{MoO}_{6} 、 \mathrm{MoO}_{3}$ 和 $(\alpha+\beta)-\mathrm{CoMoO}_{4}$ 间存在协同作用, 且这种协同作用 与 $\mathrm{MoO}_{3}$ 所占的比有关. Moens 等 ${ }^{20-22}$ 研究了铋基复 合氧化物上, 异丁烯氧化制备甲基丙烯醛反应, 结 果发现 $\mathrm{Bi}_{2} \mathrm{Sn}_{2} \mathrm{O}_{7}-\mathrm{MoO}_{3}$ 之间存在相互协同作用, 能够 明显提高反应活性. Xiong 等 ${ }^{23,24}$ 研究了 $\alpha-\mathrm{Sb}_{2} \mathrm{O}_{4}$ 和 $\mathrm{Fe}_{2}\left(\mathrm{MoO}_{4}\right)_{3}$ 上异丁烯氧化制备甲基丙烯醛反应, 纯 的 $\alpha-\mathrm{Sb}_{2} \mathrm{O}_{4}$ 上几乎没有催化活性, $\mathrm{Fe}_{2}\left(\mathrm{MoO}_{4}\right)_{3}$ 上具有 一定的催化活性但是选择性较低. 而在 $\alpha-\mathrm{Sb}_{2} \mathrm{O}_{4}$ 和 $\mathrm{Fe}_{2}\left(\mathrm{MoO}_{4}\right)_{3}$ 混合物上甲基丙烯醛的选择性和收率明 显提高. $\alpha-\mathrm{Sb}_{2} \mathrm{O}_{4}$ 为供氧体和 $\mathrm{Fe}_{2}\left(\mathrm{MoO}_{4}\right)_{3}$ 为受氧体, 它 们之间的协同作用是提高反应性能的关键. 我们课 题组对钼铋基多组分催化剂用于异丁烯选择氧化 制备甲基丙烯醛反应进行了初步的研究. ${ }^{25}$ 考察了 不同组分对催化剂的组成和性能的影响. 结果表 明, 加入适量的铋能够提高催化剂的性能. 铁和钴
元素能明显改变催化剂结构和表面性质, 从而提高 异丁烯的转化率和甲基丙烯醛的选择性. 同时发现 $\mathrm{CoMoO}_{4}$ 和新晶相以及各相间的相互作用是提高催 化剂性能的关键. 可见, 烯烃选择氧化反应的催化 剂体系种类繁多, 元素组成不同, 氧化物间的相互 作用不同, 组分间的协同作用也有明显差异. 因此 对活性中心的进一步理解有一定的研究价值和现 实意义.

本文采用共沉淀法制备了 Mo-Bi-Fe-Co-Mn 催 化剂, 考察了沉淀过程中溶液的 $\mathrm{pH}$ 值对催化剂上 各种金属氧化物的存在状态的影响, 对多组分金属 氧化物之间可能存在的协同作用进行了详细的研 究, 并与异丁烯选择氧化制备甲基丙烯醛反应结果 相关联. 从而对该反应的活性中心做了进一步的解 释和推测.

\section{2 实验部分}

\section{1 样品的制备}

Mo-Bi-Fe-Co-Mn 系列催化剂采用共沉淀法制 备. 所用试剂均为国药集团化学试剂有限公司分析 纯试剂. 一定量的钼酸铵溶于蒸馏水制得溶液 A. 称 取适量的硝酸铋溶解在硝酸水溶液中 $\left(15 \mathrm{~mol} \cdot \mathrm{L}^{-1}\right)$, 制得溶液 $\mathrm{B}$; 将一定量的铁、钴和锰的硝酸盐溶于蒸 馏水中制得溶液 $\mathrm{C}$; 溶液 $\mathrm{B}$ 和溶液 $\mathrm{C}$ 混合后得到溶 液 $\mathrm{D}$, 在 $303 \mathrm{~K}$ 搅拌的条件下把溶液 $\mathrm{D}$ 滴加到溶液 $\mathrm{A}$ 中, 用碳酸氢铵溶液调节 $\mathrm{pH}=5,6,7,8,9$, 后边摚拌 边蒸干, 干燥后的前驱物经研磨和空气气氛下 823 $\mathrm{K}$ 焙烧制得催化剂. 催化剂命名为 MB5-pH (MB5 表 示钼铋基五组分), 催化剂中 Mo:Bi:Fe:Co:Mn 的投 料比(摩尔比)为 10:0.9:1.2:4:2.6.

\section{2 样品的表征}

XRD 在 Shimazu XRD-6000 型 X 射线衍射仪上 进行, 选用 $\mathrm{Cu} K_{a}$ 辐射源, $\mathrm{Ni}$ 滤波, 管电流 $30 \mathrm{~mA}$, 管 电压 $40 \mathrm{kV}$. BET 测试在美国 Micromeritics ASAP 2010 型自动物理吸附仪上进行. 氮气为吸附质, 在 液氮温度下进行测试, 测试前试样在 $523 \mathrm{~K}$ 脱气 1 h. FTIR 在 Nicolet Impact 410 红外光谱仪上完成, $\mathrm{KBr}$ 压片, 分辨率 $2 \mathrm{~cm}^{-1}$, 测试范围为 400-2500 $\mathrm{cm}^{-1}$. XPS 采用 ESCA LAB MK II (VG Co. England ) 型 $\mathrm{X}$ 射线光电子能谱仪测试, 以 $\mathrm{Al} K_{\alpha}$ 为激发源 
(1486.6 eV), C $1 s$ 校准结合能 $284.6 \mathrm{eV} . \mathrm{H}_{2}-\mathrm{TPR}$ 在自 制的装置上进行, 样品用量 $30 \mathrm{mg}$, 还原气为 $5 \% \mathrm{H}_{2} /$ Ar混合气。

\section{3 催化反应评价}

异丁烯催化氧化制 MAL 的活性评价在固定床 流动反应器上进行, 反应器规格为 $5 \mathrm{~mm} \times 150 \mathrm{~mm}$, 反应原料气(异丁烯、氧气、氮气)的流量用质量流量 计控制, 经混和后进入反应器. 催化剂装量 $1.2 \mathrm{~mL}$, 粒度 40-60 目. 反应温度为 $633 \mathrm{~K}$, 常压下进行. 进 料空速 $\mathrm{GHSV}=3600 \mathrm{~h}^{-1}$. 反应流出物经高温六通阀 在线进样, 用 Shimadzu GC-8A 型气相色谱氢火焰 离子检测器分析.

\section{3 结果与讨论}

\section{1 催化剂的表征}

\subsection{1 催化剂的 XRD 结果}

图 1 给出 $\mathrm{MB} 5-\mathrm{pH}(\mathrm{pH}=5-9)$ 系列催化剂的 XRD结果.

与 XRD 标准谱对照, MB5-5 催化剂中有 $\mathrm{MoO}_{3}$ $\left(2 \theta=12.8^{\circ}, 23.7^{\circ}, 25.9^{\circ}, 27.3^{\circ}, 33.7^{\circ}, 39.1^{\circ}, 39.7^{\circ}\right.$, $\left.49.3^{\circ}\right)$ [JCPDS 5-508], $\mathrm{CoMoO}_{4}\left(2 \theta=14.1^{\circ}, 28.5^{\circ}\right.$, $32.3^{\circ}, 32.4^{\circ}, 25.1^{\circ}, 55.7^{\circ}$ ) [JCPDS 73-1331], $\mathrm{FeMoO}_{4}$ $\left(2 \theta=23.1^{\circ}, 26.3^{\circ}, 46.6^{\circ}, 53.3^{\circ}, 58.4^{\circ}\right)$ [JCPDS 28 0488], $\mathrm{Bi}_{2}\left[\mathrm{MoO}_{6}\right]\left(2 \theta=27.4^{\circ}, 31.0^{\circ}, 27.3^{\circ}, 31.9^{\circ}\right)$ [JCPDS 82-2067] 和 $\mathrm{Bi}_{2} \mathrm{Mo}_{3} \mathrm{O}_{12}\left(2 \theta=27.9^{\circ}, 28.0^{\circ}\right.$, $29.2^{\circ}, 31.0^{\circ}, 36.1^{\circ}$ ) [JCPDS 78-2420] 晶相的衍射峰. 当 $\mathrm{pH}=7$ 时, 催化剂的 XRD 谱中各氧化物相的衍射 峰变弱. 当 $\mathrm{pH}=8,9$ 时, 各氧化物的衍射峰明显增 强. 表明 $\mathrm{pH}=7$ 的催化剂上各组分间存在某种相互 作用, 这种相互作用在某种程度上可提高各组分的

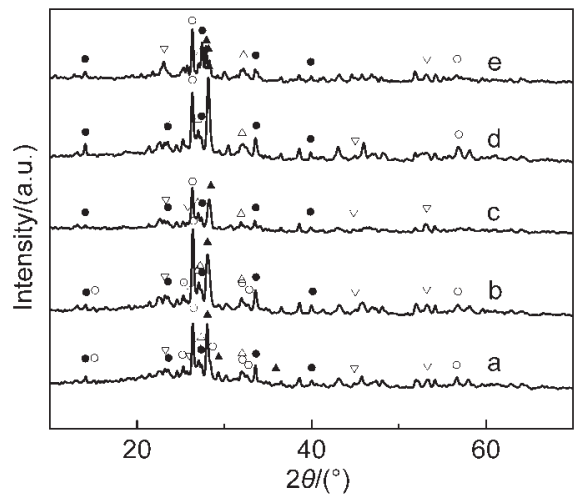

图 1 MB5-pH催化剂的 XRD 谱图

Fig.1 XRD patterns of MB5-pH catalysts

$(\bullet) \mathrm{MoO}_{3},(\circ) \mathrm{CoMoO}_{4},(\nabla) \mathrm{FeMoO}_{4},(\triangle) \mathrm{Bi}_{2}\left[\mathrm{MoO}_{6}\right]$,

(ム) $\mathrm{Bi}_{2} \mathrm{Mo}_{3} \mathrm{O}_{12}$, crystalline phase; $\mathrm{pH}$ : (a) 5, (b) 6, (c) 7, (d) 8, (e) 9
分散程度或减小了物相的粒径. XRD 的研究结果表 明, 由于沉淀母液中的 $\mathrm{pH}$ 值不同可能影响各组分 的沉淀过程, 从而影响焙烧后催化剂中的各组分间 的作用, 导致最终催化剂中物相的分散程度存在差 异.

\section{1 .2 催化剂的FTIR 表征}

MB5-pH (pH=5-9)系列催化剂的红外结果见 图 2. 由图可知, 在 $993 、 870$ 和 $820 \mathrm{~cm}^{-1}$ 处的振动峰 可归属于 $\mathrm{Mo}=\mathrm{O}$ 的键伸缩振动, ${ }^{15}$ 表明催化剂中有 $\mathrm{MoO}_{3}$ 存在. $412 \mathrm{~cm}^{-1}$ 处的振动峰归属于 $\mathrm{Fe}-\mathrm{O}$ 的振 动. ${ }^{25} 947 \mathrm{~cm}^{-1}$ 处的振动峰为 $\mathrm{Fe}-\mathrm{O}-\mathrm{Mo}$ 的特征振 动, 847 和 $845 \mathrm{~cm}^{-1}$ 处的振动峰归于 $\mathrm{Mo}-\mathrm{O}$ 单键的 振动, ${ }^{26}$ 也有研究者将 $847 \mathrm{~cm}^{-1}$ 处的振动归于 $\mathrm{Bi}$ 融入 $\mathrm{Fe}-\mathrm{O}-\mathrm{Mo}$ 形成的某种复合氧化物的振动峰. ${ }^{27}$ 在 848 、784 和 $656 \mathrm{~cm}^{-1}$ 处出现的特征峰可归于 $\mathrm{Co}-$ $\mathrm{O}-\mathrm{Mo}$ 的特征峰. ${ }^{28}$ 随着 $\mathrm{pH}$ 值的改变, $947 、 848$ 和 $656 \mathrm{~cm}^{-1}$ 处的振动峰有显著的变化. $\mathrm{pH}=7$ 的催化剂 在 938、947、848 和 $656 \mathrm{~cm}^{-1}$ 等处的振动峰与其它催 化剂相比明显变弱. 表明催化剂上各氧化物间的键 能发生一定的改变. 这是由于各种金属和氧间的作 用发生了改变, 导致部分氧化物的 $\mathrm{M}-\mathrm{O}$ 的振动减 弱.

\subsection{3 催化剂的 XPS 表征}

焙烧处理后的 MB5- $\mathrm{pH}(\mathrm{pH}=5-9)$ 系列催化剂 的 XPS 表征结果见图 3 和表 1 . 由图 3 和表 1 结果可 知, 随着 $\mathrm{pH}$ 值的逐渐提高, $\mathrm{Mo}$ 的结合能逐渐降低, $\mathrm{pH}=7$ 时其结合能为 $232.4 \mathrm{eV}$, 此后随 $\mathrm{pH}$ 值继续提 高, $\mathrm{Mo}$ 的结合能增加, 当 $\mathrm{pH}=9$ 时其结合能为 232.8 $\mathrm{eV} .^{15,24}$ 同时, 由 Mo $3 d$ 的能谱可见有肩峰存在, 表明 催化剂表面含有一定量的 $\mathrm{Mo}^{5+}$ 物种. 这一结论与 $\mathrm{XRD}$ 结果(催化剂中含有 $\mathrm{FeMoO}_{4}$ 物种)相对应. $\mathrm{pH}=$

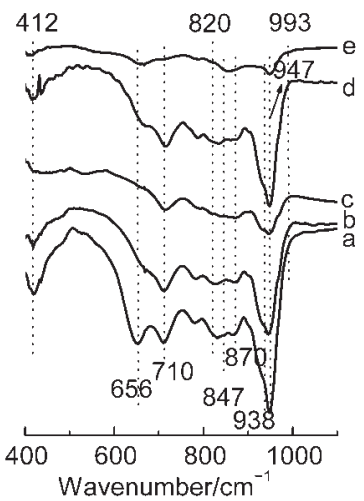

图 2 MB5-pH催化剂的 FTIR 谱图

Fig.2 FTIR spectra of MB5-pH catalysts pH: (a) 5, (b) 6, (c) 7, (d) 8, (e) 9 

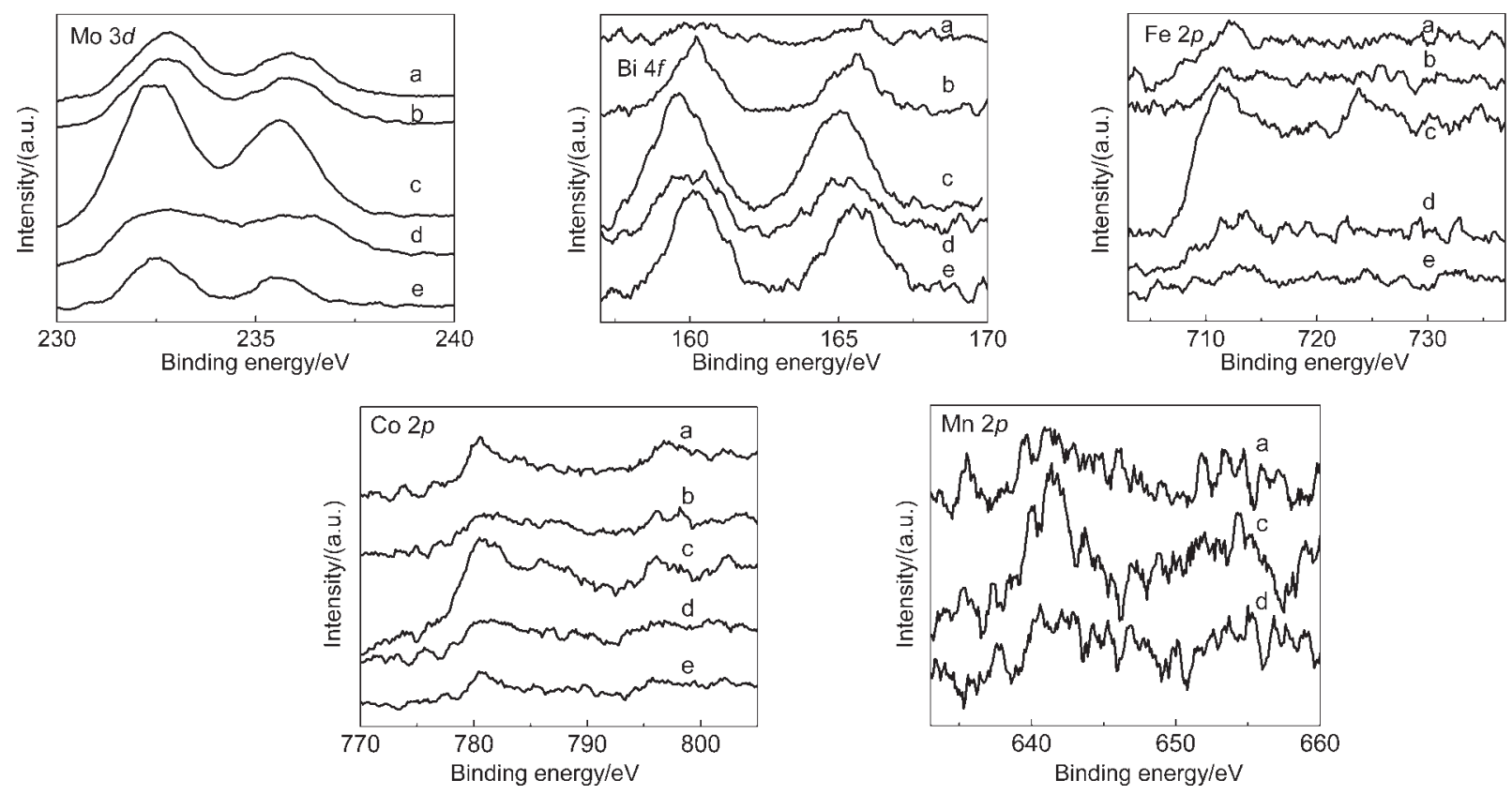

图 3 MB5-pH 催化剂的XPS 谱图

Fig.3 XPS spectra of MB5-pH catalysts

pH: (a) 5, (b) 6, (c) 7, (d) 8, (e) 9

7 的催化剂表面 $\mathrm{Bi}$ 的结合能为 $159.6 \mathrm{eV} .{ }^{19}$ 除此之 外, 其它催化剂表面 $\mathrm{Bi}$ 的结合能基本没有变化, 均 为 $160.0 \mathrm{eV}$ 左右. 从表中可知, $\mathrm{Fe}$ 的结合能也有很 大变化, $\mathrm{pH}=7$ 的催化剂表面 $\mathrm{Fe}$ 的结合能最低, 仅为 $711.3 \mathrm{eV}$. 其它催化剂表面 $\mathrm{Fe}$ 的结合能均大于 711.3 $\mathrm{eV}^{24} \mathrm{pH}$ 值对 $\mathrm{Co}$ 的结合能的影响没有明显规律. $\mathrm{pH}=7$ 的催化剂表面 $\mathrm{Mn}$ 的结合能为 $641.4 \mathrm{eV}$, 而 $\mathrm{pH}$ 值的变化对其它催化剂表面 $\mathrm{Mn}$ 的结合能基本没有 影响. 在 $\mathrm{pH}=6,9$ 的催化剂中没有检测到 $\mathrm{Mn}$ 的存 在, 可能是在这两个催化剂表面 $\mathrm{Mn}$ 的量低于检测 限. XPS 的研究结果表明, 在催化剂表面上, Mo 元 素主要以 $\mathrm{Mo}^{6+}, \mathrm{Bi}$ 元素以 $\mathrm{Bi}^{3+}, \mathrm{Fe}$ 元素以 $\mathrm{Fe}^{3+}, \mathrm{Co}$ 元 素以 $\mathrm{Co}^{2+}, \mathrm{Mn}$ 元素以 $\mathrm{Mn}^{2+}$ 的价态形式存在. 这些数 据基本与 XRD 的表征结果相符. 但是 $\mathrm{pH}=7$ 的催化 剂表面 $\mathrm{Mo} 、 \mathrm{Bi} 、 \mathrm{Fe} 、 \mathrm{Co}$ 四种元素的结合能都比其它 的小, 表明在该催化剂表面形成了一种键能较小的
物相, 这与氧化物间的相互作用有关.

催化剂表面的元素组成见表 1 . 与其它催化剂 相比, $\mathrm{pH}=7$ 的催化剂表面各元素的含量均相对较 少, 进一步说明合成过程中 $\mathrm{pH}$ 值对催化剂的元素 组成有较大影响.

\section{1 .4 催化剂的BET 结果}

MB5-pH 催化剂的 BET 表征结果见表 1. 从表 中可以看出, 沉淀母液的 $\mathrm{pH}$ 值对催化剂的比表面 有明显的影响. 当 $\mathrm{pH}=7$ 时比表面最大为 $3.6 \mathrm{~m}^{2} \cdot \mathrm{g}^{-1}$. 其它催化剂的比表面均小于 $2.0 \mathrm{~m}^{2} \cdot \mathrm{g}^{-1}$. 表明 $\mathrm{pH}$ 值 对催化剂的表面结构有明显的影响, 合适的 $\mathrm{pH}$ 值 能够有效的增加催化剂的比表面, 即能够有效的增 加活性中心的数量.

\subsection{5 催化剂的 $\mathrm{H}_{2}-\mathrm{TPR}$ 结果}

焙烧处理后的 MB5-pH 系列催化剂的 $\mathrm{H}_{2}$-TPR 表征结果见图 4 和表 2 . 在多组分氧化物或复合氧化

表 1 MB5-pH催化剂的 BET 和 XPS 结果

Table 1 BET surface area and XPS data of MB5-pH catalysts

\begin{tabular}{|c|c|c|c|c|c|c|c|}
\hline \multirow{2}{*}{$\mathrm{pH}$} & \multirow{2}{*}{$S_{\mathrm{BET}} /\left(\mathrm{m}^{2} \cdot \mathrm{g}^{-1}\right)$} & \multirow{2}{*}{ Surface composition of $\mathrm{Mo} / \mathrm{Bi} / \mathrm{Fe} / \mathrm{Co} / \mathrm{Mn}^{\mathrm{a}}$} & \multicolumn{5}{|c|}{ Binding energy/eV } \\
\hline & & & $\operatorname{Mo~} 3 d_{5 / 2}$ & $\operatorname{Bi} 4 f_{7 / 2}$ & $\mathrm{Fe} 2 p_{3 / 2}$ & $\mathrm{Co} 2 p_{3 / 2}$ & $\operatorname{Mn} 2 p_{3 / 2}$ \\
\hline 5 & 1.8 & $1: 0.06: 0.35: 0.88: 0.21$ & 232.8 & 160.2 & 711.6 & 780.55 & 641.2 \\
\hline 6 & 1.9 & $1: 0.10: 0.37: 1.56: 0$ & 232.7 & 160.1 & 712.1 & 781.2 & - \\
\hline 7 & 3.6 & 1:0.09:0.33:0.46:0.12 & 232.4 & 159.6 & 711.3 & 780.8 & 641.4 \\
\hline 8 & 1.9 & $1: 0.19: 0.72: 1.10: 0.34$ & 232.5 & 160.0 & 712.4 & 781.2 & 641.2 \\
\hline 9 & 0.4 & $1: 0.17: 0.52: 0.64: 0$ & 232.8 & 160.1 & 713.0 & 780.9 & - \\
\hline
\end{tabular}


物的 $\mathrm{H}_{2}-\mathrm{TPR}$ 的研究中, 由于复合氧化物之间的协同 效应, $\mathrm{H}_{2}$-TPR 中单个还原峰很难归属为单一氧化物 的还原. ${ }^{29}$ 因此还原峰可能是某种金属的氧化物或 多金属复合氧化物的共同还原的结果, 也可能高价 氧化物分步还原到低价态的结果. ${ }^{30}$ 在我们前期研 究工作中, 钼基金属氧化物体系的 $\mathrm{H}_{2}$-TPR 进行了研 究, 结果发现 Mo-Bi、Mo-Fe 和 Mo-Co 等复合氧化物 的还原峰均在 923-1025 K 范围内, 即高温还原峰可 认为主要是 $\mathrm{CoMoO}_{4}$ 和 $\mathrm{FeMoO}_{4}$ (包括部分钿铋物 种)的还原. ${ }^{25}$

在本文中, $\mathrm{pH}=5$ 时催化剂在 $722 、 755$ 和 $793 \mathrm{~K}$ 处 均有还原峰, 可归属为不同晶粒的 $\mathrm{MoO}_{3}$ 的还原. XRD、FTIR 和 XPS 的研究结果表明, $\mathrm{pH}=5$ 的催化剂 表面有 $\mathrm{MoO}_{3}$ 存在. $\mathrm{pH}>5$ 的催化剂上这三个还原峰 明显减小 (甚至消失), 说明在 $\mathrm{pH}>5$ 的催化剂上 $\mathrm{MoO}_{3}$ 物种减少. 这与 FTIR 的研究结果基本相符. 图 中 873-1073 K 间的还原峰可归结为 $\mathrm{CoMoO}_{4}$ 、 $\mathrm{FeMoO}_{4} 、 \mathrm{Bi}_{2}\left[\mathrm{MoO}_{6}\right]$ 和 $\mathrm{Bi}_{2} \mathrm{Mo}_{3} \mathrm{O}_{12}$ 等复合氧化物的还 原或各组分间相互作用共同还原的结果. 由图 4 和 表 2 可知, $\mathrm{pH}=6,7,8$ 催化剂的 $\mathrm{H}_{2}$-TPR 谱在 $838 \mathrm{~K}$ 附 近都有一个还原峰. 其中 $\mathrm{pH}=7$ 催化剂该物相的还 原峰面积最大(表 2), $\mathrm{pH}=6,8$ 的催化剂在 $838 \mathrm{~K}$ 处的 还原峰较小. XRD 的研究结果表明 $\mathrm{pH}=7$ 的催化剂 上各氧化物相的衍射峰均较弱. 可见的明显物相衍

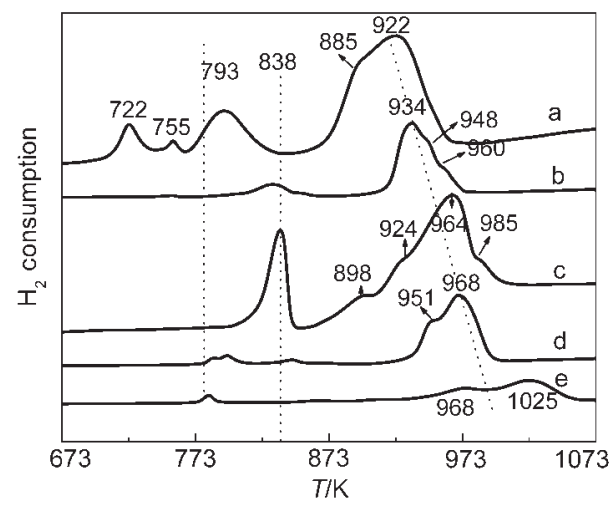

图 4 MB5-pH 催化剂的 $\mathrm{H}_{2}$-TPR 谱图

Fig.4 $\mathrm{H}_{2}$-TPR results of $\mathrm{MB5}-\mathrm{pH}$ catalysts pH: (a) 5, (b) 6, (c) 7, (d) 8, (e) 9

表 2 MB5-pH催化剂的 $\mathrm{H}_{2}$-TPR 数据 Table $2 \mathrm{H}_{2}$-TPR data of MB5-pH catalysts

\begin{tabular}{clcl}
\hline $\mathrm{pH}$ & \multicolumn{1}{c}{$T / \mathrm{K}$} & $m / \mathrm{mg}$ & \multicolumn{1}{c}{$\mathrm{H}_{2}$ consumption $/ \mu \mathrm{mol}$} \\
\hline 5 & $722 / 755 / 793 / 885 / 922$ & 50 & $1504 / 526 / 3676 / 1329 / 14936$ \\
6 & $831 / 934 / 948 / 960$ & 50 & $1557 / 2477 / 131 / 2759$ \\
7 & $838 / 898 / 924 / 964 / 985$ & 50 & $3854 / 843 / 1415 / 11850 / 561$ \\
8 & $794 / 844 / 951 / 968$ & 50 & $288 / 74 / 1266 / 3780$ \\
9 & $784 / 858 / 968 / 1025$ & 50 & $150 / 199 / 1439 / 1478$ \\
\hline
\end{tabular}

射峰为 $\mathrm{CoMoO}_{4}$ 和 $\mathrm{Bi}_{2} \mathrm{Mo}_{3} \mathrm{O}_{12}$, 对比 $\mathrm{pH}=5,6,8,9$ 的 XRD 和其 $\mathrm{H}_{2}-\mathrm{TPR}$ 的研究结果, $838 \mathrm{~K}$ 处的还原峰不 能归结为 $\mathrm{CoMoO}_{4}$ 和 $\mathrm{Bi}_{2} \mathrm{Mo}_{3} \mathrm{O}_{12}$ 等氧化物的还原. 因 此, 我们将该还原峰对应的特定物相命名为 MX相. FTIR 的研究结果表明, 在 $\mathrm{pH}=7$ 催化剂上, 各种金属 和氧间的作用发生了改变, 导致部分氧化物的 $\mathrm{M}-$ $\mathrm{O}$ 的振动减弱. XPS 的研究结果也证明了 $\mathrm{pH}=7$ 的催 化剂表面 Mo、Bi、Fe、Co 四种元素的结合能都比其 它的小. 因此, $838 \mathrm{~K}$ 处的还原峰与催化剂中某种特 定相 MX 的还原有确切的关联性. 由于组分多, 催化 剂上的各氧化物组分间存在复杂的相互作用, 因此 该物种无法确定其确切组成. 我们推测 $838 \mathrm{~K}$ 处的 还原峰为某种特定相中的几种金属复合氧化物共 同还原的结果. $\mathrm{pH}=6,8$ 催化剂的 $\mathrm{H}_{2}-\mathrm{TPR}$ 的谱图中 也可见 $838 \mathrm{~K}$ 左右的还原峰, 其峰面积较 $\mathrm{pH}=7$ 催化 剂的小. $\mathrm{H}_{2}-\mathrm{TPR}$ 的研究结果表明, 溶液的 $\mathrm{pH}$ 值确实 影响催化剂中金属氧化物的存在状态和形式.

\section{2 催化剂反应活性结果}

图 5 给出共沉淀法制备的 MB5-pH ( $\mathrm{pH}=5-9)$ 系 列催化剂性能考察结果. 由图可见, $633 \mathrm{~K}$ 反应时, 随着 $\mathrm{pH}$ 值不断升高, 异丁烯的转化率和 MAL 的选 择性逐渐升高. $\mathrm{pH}=7$ 时, 异丁烯的转化率和 MAL 的 选择性均达到最大值, 分别为 $99.9 \%$ 和 $95.6 \%$. 继续 提高 $\mathrm{pH}$ 值, 异丁烯的转化率和 MAL 的选择性逐渐 降低. 其它产物如 MAA、醋酸(HAc)、丙酮和碳的氧 化物的含量均很低, $\mathrm{pH}=7$ 时碳的氧化物的选择性最 低. 继续提高 $\mathrm{pH}$ 值, 碳的氧化物选择性略有提高.

从反应结果上看, $\mathrm{pH}=5$ 的催化剂上异丁烯的转 化率约为 $24.3 \%$ 左右. XRD 和 $\mathrm{H}_{2}$-TPR 结果证明该催

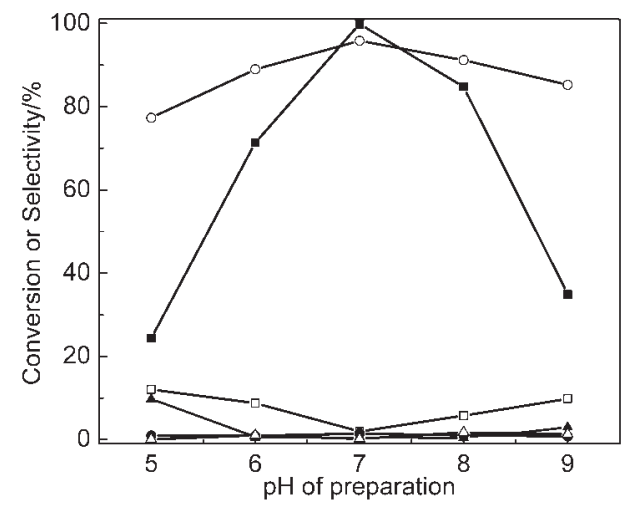

图 5 MB5-pH系列催化剂的活性测试结果

Fig.5 Catalytic results of MB5-pH catalysts

gas hourly space velocity (GHSV): $3600 \mathrm{~h}^{-1}$, calcinations temperature: $823 \mathrm{~K}$, reaction time: $3-4 \mathrm{~h}$. (®) conversion ofisobutene, (口) selectivity of $\mathrm{CO}_{x},(\mathbf{\Lambda})$ selectivity of $\mathrm{HAc},(\triangle)$ selectivity of MAA, (०) selectivity of MAL, $(\bullet)$ selectivity of acetone 
化剂上主要以 $\mathrm{MoO}_{3} 、 \mathrm{CoMoO}_{4} 、 \mathrm{FeMoO}_{4} 、 \mathrm{Bi}_{2}\left[\mathrm{MoO}_{6}\right]$ 和 $\mathrm{Bi}_{2} \mathrm{Mo}_{3} \mathrm{O}_{12}$ 等物种存在. 表明三氧化钼和钿的钴、 铁和铋的复合氧化物对异丁烷的活化能力不强, 而 副产物中碳氧化合物的选择性较高, 表明 $\mathrm{MoO}_{3}$ 有 很强的氧化能力, 深度氧化产物较多. Gaigneaux ${ }^{7}$ 和 Guan ${ }^{8}$ 等研究钼基多金属催化剂上异丁烯选择氧化 反应, 发现 $\mathrm{MoO}_{3}$ 催化剂上异丁烯的转化率和甲基 丙烯醛的选择性均不高, 产物中有大量的碳氧化合 物. 随着 $\mathrm{pH}$ 值进一步提高, 当 $\mathrm{pH}=6$ 时, $\mathrm{H}_{2}-\mathrm{TPR}$ 结果 表明催化剂中开始含有 MX物种, 此时异丁烯的转 化率明显提高为 $71.4 \%$, 而甲基丙烯醛的选择性可 达到 $89.0 \% . \mathrm{pH}=7$ 时, 催化剂上 MX物种的量最大, 此时, 异丁烯的转化率和 MAL 的选择性均达到最 大值, 分别为 $99.9 \%$ 和 $95.6 \%$. 当 $\mathrm{pH}=8$ 时, 催化剂上 有少量的 MX物种, 异丁烯的转化率和甲基丙烯醛 的选择性相对降低. 催化剂的活性测试结果和催化 剂上物种组成有一定的关联性, 即 MX物种对异丁 烯的转化率和甲基丙烯醛的选择性影响较大. 可以 认为 MX物种是反应的主要活性物种之一. 当然, 催 化剂上各物种间的相互协同作用对异丁烯选择氧 化反应也有重要作用. ${ }^{7,19-23}$

\section{4 结 论}

考察了 $\mathrm{pH}$ 值对钼铋基多组分催化剂性能的影 响, 发现沉淀过程中的 $\mathrm{pH}$ 值不同, 焙烧后催化剂上 的物相组成不同. 催化剂上 MX 物种的量随 $\mathrm{pH}$ 值的 改变而变化, 异丁烯的转化率和甲基丙烯醛的选择 性与 MB5-pH 催化剂上 MX 物种的量有一定的对应 关系. 各物种间的相互协同作用对异丁烯选择氧化 反应也有重要作用. $\mathrm{pH}=7$ 的催化剂反应性能最佳, 异丁烯的转化率和 MAL 的选择性分别为 $99.9 \%$ 和 $95.6 \%$.

\section{References}

(1) Zhu, W. C.; Jia, M. J.; Wang, Z. L.; Wang, G. J.; Wu, T. H. Chem. J. Chin. Univ. 2007, 28 (2), 334 . [ 朱万春, 贾明君, 王振旅, 王国甲, 吴通好. 高等学校化学学报, 2007, 28 (2), 334.]

(2) Ono, T.; Utsumi, K.; Kataoka, M.; Tanaka, Y.; Noguchi, F. Catal. Today 2004, 91-92, 181.

(3) Liu, H.; Gaigneaux, E. M.; Imoto, H.; Shido, T.; Iwasawa, Y. Appl. Catal. A 2000, 202, 251. doi: 10.1016/S0926-860X(00) 00539-1

(4) Inoue, T.; Oyama, S. T.; Imoto, H.; Asakura, K.; Iwasawa, Y.
Appl. Catal. A2000, 191, 131. doi: 10.1016/S0926-860X(99) 00314-2

(5) Cai, T. J.; Yu, C. L.; Deng, Q.; Peng, Z. S.; Long, Y. F.; Jiang, S. L. Chin. J. Catal. 2003, 24, 951. [蔡铁军, 余长林, 邓 谦, 彭振山, 龙云飞, 蒋绍亮. 催化学报, 2003, 24, 951.]

(6) Song, N.; Rhodes, C.; Bartley, J. K.; Taylor, S. H.; Chadwick, D.; Hutchings, G. J. J.Catal. 2005, 236, 282. doi: 10.1016/ j.jcat.2005.10.008

(7) Gaigneaux, E. M.; Genet, M. J.; Ruiz, P.; Delmon, B. J. Phys. Chem. B 2000, 104, 5724. doi: 10.1021/jp9913416

(8) Guan, J. Q.; Xu, C.; Wang, Z. Q.; Yang, Y.; Liu, B.; Shang, F. P.; Shao, Y. Q.; Kan, Q. B. Catal. Lett. 2008, 124, 428. doi: 10.1007/s10562-008-9496-3

(9) Wang, L.; Li, Z. X.; Zhang, S. J.; Zhang, X. P. The Chinese Journal of Process Engineering 2007, 7 (1), 202. [王＼cjkstart蕾, 李增喜, 张锁江, 张香平. 过程工程学报, 2007, 7 (1), 202.]

(10) Grzybowska, B.; Haber, J.; Komorek, J. J. Catal. 1972, $25,25$. doi: 10.1016/0021-9517(72)90198-4

(11) Wang, X. T.; Bi, C.; Zhong, S. H.; Xiao, X. F. Chin. J. Catal. 2010, 31, 84. [王希涛, 毕 超, 钟顺和, 肖秀芬. 催化学报, 2010, 31, 84.] doi: 10.3724/SP.J.1088.2010.90634

(12) Jung, J. C.; Lee, H.; Kim, H.; Chung, Y. M.; Kim, T. J.; Lee, S. J.; Oh, S. H.; Kim, Y. S.; Song, I. K.Catal. Commun. 2008, 9, 1676. doi: 10.1016/j.catcom.2008.01.026

(13) Jung, J. C.; Lee, H.; Seo, J. G.; Seo, J. G.; Park, S.; Chung, Y. M.; Kim, T. J.; Lee, S. J.; Oh, S. H.; Kim, Y. S.; Song, I. K. Catal. Today 2009, 141, 325.

(14) Jung, J. C.; Lee, H.; Kim, H.; Park, S.; Chung, Y. M.; Kim, T. J.; Lee, S. J.; Oh, S. H.; Kim, Y. S.; Song, I. K. Catal. Commun. 2008, 9, 2059. doi: 10.1016/j.catcom.2008.03.052

(15) Guan, J. Q.; Wu, S. J.; Wang, H. S.; Jing, S. B.; Wang, G. J.; Zhen, K. J.; Kan, Q. B. J. Catal. 2007, 251, 354. doi: 10.1016/ j.jcat.2007.07.028

(16) Van Well, W. J. M.; Le, M. T.; Schiødt, N. C.; Hoste, S.; Stoltze, P. J. Mol. Catal. A 2006, 256, 1. doi: 10.1016/j.molcata. 2006.04.030

(17) Jo, B. Y.; Kim, E. J.; Moon, S. H. Appl. Catal. A 2007, 332, 257. doi: 10.1016/j.apcata.2007.08.025

(18) Le, M. T.; Van Well, W. J. M.; Stoltze, P.; Driessche, I. V.; Hoste, S. Appl. Catal. A 2005, 282, 189. doi: 10.1016/j.apcata. 2004.12.010

(19) Carrazán, S. R. G.; Martín, C.; Rives, V.; Vidal, R. Appl. Catal. $A$ 1996, 135, 95. doi: 10.1016/0926-860X(95)00226-X

(20) Moens, L.; Ruiz, P.; Delmon, B.; Devillers, M. Appl. Catal. A 1998, 171, 131. doi: 10.1016/S0926-860X(98)00075-1

(21) Moens, L.; Ruiz, P.; Delmon, B.; Devillers, M. Appl. Catal. A 1999, 180, 299. doi: 10.1016/S0926-860X(98)00360-3

(22) Moens, L.; Ruiz, P.; Delmon, B.; Devillers, M. Appl. Catal. A 2003, 249, 365. doi: 10.1016/S0926-860X(03)00225-4

(23) Xiong, Y. L.; Weng, L. T.; Bertrand, P.; Ladrière, J.; Daza, L.; Ruiz, P.; Delmon, B. J. Mol. Catal. A 2000, 155, 59. doi: 
10.1016/S1381-1169(99)00319-2

(24) Xiong, Y. L.; Cadus, L. E.; Daza, L.; Bertrand, P.; Ladrière, J.; Ruiz, P.; Delmon, B. Topics Catal. 2000, 11/12, 167.

(25) Qu, Y. L.; Liu, S. Y.; Wang, Z. L.; Xu, J. N. Chem. J. Chin. Univ. 2010, 31 (2), 325. [曲滟立, 刘士艳, 王振旅, 徐家宁. 高等学校化学学报, 2010, 31 (2), 325.]

(26) Li, J.; Song, W.; Dou, B. S. Chin. J. Appl. Chem. 1996, 13 (5), 58. [李 静, 宋 伟, 窦伯生. 应用化学, 1996, 13 (5), 58.]

(27) Sun, R. Q.; Gu, X. Y.; Zhang, H. H.; Yang, R. S.; Yang, Q. Y.; Zhao, S. L. Spectroscopy and Spectral Analysis 2000, 20 (6), 857. [孙瑞卿, 喜晓燕, 张汉辉, 杨融生, 杨齐愉, 赵士龙.
光谱学与光谱分析, 2000, 20 (6), 857.]

(28) Wang, G. J.; Wu, T. H.; Yang, H. M.; Jiang, Y. Z.; Ji, Y. S. Chem. J. Chin. Univ. 1994, 15 (12), 1845. [王国甲, 吴通好, 杨洪茂, 姜玉子, 稽玉书. 高等学校化学学报, 1994, 15 (12), 1845.]

(29) Cai, T. J.; Deng, Q.; Huang, D. F.; Peng, Z. S.; Long, Y. F.; Chen, Y. Z. Chin. J. Inorg. Chem. 2002, 18 (6), 602. [蔡铁军, 邓 谦, 黄德发, 彭振山, 龙云飞, 陈亚中. 无机化学学报, 2002, 18 (6), 602.]

(30) Ma, W. P.; Kugler, E. L.; Wright, J.; Dadyburjor, D. B. Energy \& Fuels 2006, 20, 2299. doi: 10.1021/ef0602372 